Published in final edited form as:

ACS Biomater Sci Eng. 2016 May 9; 2(5): 829-837. doi:10.1021/acsbiomaterials.6b00060.

\title{
Multiple Administrations of Viral Nanoparticles Alter in Vivo Behavior-Insights from Intravital Microscopy
}

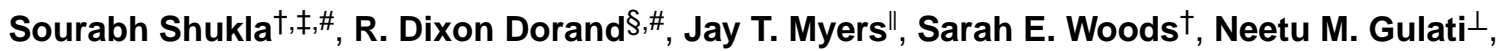 \\ Phoebe L. Stewart ${ }^{\perp}$, Ulrich Commandeur ${ }^{\star}$, Alex Y. Huang ${ }^{\ddagger}, \S, \|,{ }^{*}$, and Nicole F.

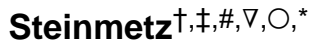 \\ †Department of Biomedical Engineering, Case Western Reserve University, 10900 Euclid Avenue, \\ Cleveland, Ohio 44106, United States \\ ‡Department of Case Comprehensive Cancer Center, Case Western Reserve University, 10900 \\ Euclid Avenue, Cleveland, Ohio 44106, United States \\ $\S$ Department of Pathology, Case Western Reserve University, 10900 Euclid Avenue, Cleveland, \\ Ohio 44106, United States \\ "Department of Pediatrics, Case Western Reserve University, 10900 Euclid Avenue, Cleveland, \\ Ohio 44106, United States \\ ${ }^{\perp}$ Department of Pharmacology, and Cleveland Center for Membrane and Structural Biology, Case \\ Western Reserve University, 10900 Euclid Avenue, Cleveland, Ohio 44106, United States \\ \#Department of Radiology, Case Western Reserve University, 10900 Euclid Avenue, Cleveland, \\ Ohio 44106, United States \\ ${ }^{\nabla}$ Department of Macromolecular Science and Engineering, Case Western Reserve University, \\ 10900 Euclid Avenue, Cleveland, Ohio 44106, United States \\ Department of Materials Science and Engineering, Case Western Reserve University, 10900 \\ Euclid Avenue, Cleveland, Ohio 44106, United States \\ -Institute for Molecular Biotechnology, RWTH Aachen University, Worringer Weg 1, 52074 \\ Aachen, Germany
}

\section{Abstract}

\footnotetext{
*Corresponding Authors. alex.y.huang@case.edu. nicole.steinmetz@case.edu. \#These authors have contributed equally. ASSOCIATED CONTENT

Supporting Information

The Supporting Information is available free of charge on the ACS Publications website at DOI: 10.1021/acsbiomaterials.6b00060.
}

C57BL/6-Tg(UBC-GFP) mice vasculature imaged as nonfluorescent channel surrounded by GFP expressing surrounding tissues (Figure S1); intravital imaging performed over 10 min postinjection of PVX-A647 particles upon the three weekly i.v. administrations (Figure S2); and snapshots from imaging data showing lack of significant colocalization of PVX-A647 with cells in the vasculature (Figure S3) (PDF)

Intravital imaging performed over 10 min postinjection of PVX-A647 particles upon the three weekly i.v. administrations (AVI) 
Multiple administrations of nanoparticle-based formulations are often a clinical requirement for drug delivery and diagnostic imaging applications. Steady pharmacokinetics of nanoparticles is desirable to achieve efficient therapeutic or diagnostic outcomes over such repeat administrations. While clearance through mononuclear phagocytic system is a key determinant of nanoparticle persistence in vivo, multiple administrations could potentially result in altered pharmacokinetics by evoking innate or adaptive immune responses. Plant viral nanoparticles (VNPs) represent an emerging class of programmable nanoparticle platform technologies that offer a highly organized proteinaceous architecture and multivalency for delivery of large payloads of drugs and molecular contrast agents. These very structural features also render them susceptible to immune recognition and subsequent accelerated systemic clearance that could potentially affect overall efficiency. While the biodistribution and pharmacokinetics of VNPs have been reported, the biological response following repeat administrations remains an understudied area of investigation. Here, we demonstrate that weekly administration of filamentous plant viruses results in the generation of increasing levels of circulating, carrier-specific $\operatorname{IgM}$ and $\operatorname{IgG}$ antibodies. Furthermore, PVX specific immunoglobulins from the serum of immunized animals quickly form aggregates when incubated with PVX in vitro. Such aggregates of VNP-immune complexes are also observed in the mouse vasculature in vivo following repeat injections when imaged in real time using intravital two-photon laser scanning microscopy (2P-LSM). The size of aggregates diminishes at later time points, coinciding with antibody class switching from IgM to IgG. Together, our results highlight the need for careful in vivo assessment of (viral) nanoparticle-based platform technologies, especially in studying their performance after repeat administration. We also demonstrate the utility of intravital microscopy to aid in this evaluation.

\section{Graphical abstract}

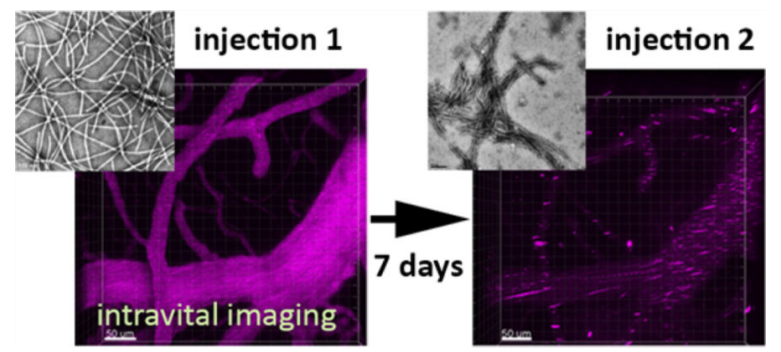

\section{Keywords}

viral nanoparticles; repeat administration; intravital imaging; nanoparticle circulation; antibody response; IgM antibody

\section{INTRODUCTION}

Nanoparticle-based therapeutic and diagnostic approaches are rapidly evolving for use in the clinic for early diagnosis and treatment of cancer, cardiovascular diseases, and infectious diseases. ${ }^{1}$ Nanoparticles can carry and deliver high payloads of therapeutic molecules or contrast agents to targeted tissues and cells, thereby minimizing off-target effects while improving the solubility and in vivo stability of payloads. ${ }^{1,2}$ The efficiency of such nanoformulations depends on their ability to surmount numerous physiological barriers 
including rapid clearance by cells of the reticuloendothelial system (RES) that can adversely affect nanoparticle pharmacokinetics and targeting. ${ }^{3,4}$ Thus, careful evaluation of the in vivo behavior, biological fate, and long-term safety of nanoparticle-based platforms is a key requirement toward their successful clinical translation. ${ }^{5,6}$ Adsorption of plasma proteins on the nanoparticle surface in vivo dictates the accumulation of nanoparticles by cells of RES. This process of opsonization tags the nanocarrier for sequestration in mononuclear phagocyte cells such as neutrophils and monocytes, triggering immune activation. ${ }^{7,8}$ The extent and variety of adsorbed proteins varies from one type of nanoparticle to another and is a function of surface properties, shape, size, and charge of the nanoparticles. ${ }^{3}$

Viral nanoparticles (VNPs) derived from plant viruses or bacteriophages have emerged as a highly versatile platform technology for various biomedical applications. ${ }^{9-11}$ These proteinaceous nanoparticles offer structural and functional programmability through genetic and/or chemical engineering methods. ${ }^{12}$ The highly organized and multivalent VNP architecture is an excellent platform for delivery of large payloads of drugs and contrast agents. These structural features also render VNPs highly susceptible to immune recognition leading to the generation of carrier specific antibodies. ${ }^{13-15}$ The immunostimulatory potential of VNPs makes them attractive platforms for vaccine applications ${ }^{13,16}$ and immunotherapies. ${ }^{17}$ However, this property is a double-edged sword; the inherent immunogenicity may be an undesirable attribute for drug delivery and imaging applications. Recognition and neutralization by antibodies could alter the in vivo fate of VNPs, leading to enhanced clearance and deposition in nontarget tissues. In fact, the presence of neutralizing antibodies and antibody-mediated clearance of viral gene delivery platforms, based on mammalian viruses, are the biggest obstacles for their clinical success and have limited their application to localized administrations. ${ }^{18}$

Chemical ligation strategies to stealth and camouflage nanoparticles have been developed to overcome this; for example, we recently demonstrated that VNPs escape antibody recognition through coating with PEG, ${ }^{19}$ silica, ${ }^{20}$ or serum proteins.${ }^{21}$ Nevertheless, the effects of carrier-specific interactions after repeat administration of plant-based VNPs have not yet been studied. To fill this gap, we set out to evaluate the in vivo fates of filamentous plant virus-based carriers, specifically Potato virus X (PVX), after repeat administration.

PVX is a plant-virus-derived flexible filamentous nanoparticle measuring $515 \times 13 \mathrm{~nm}$ comprised of 1270 identical copies of a single coat protein. PVX offers excellent structural and functional programmability through genetic and chemical engineering strategies for a range of biomedical applications. Given the helical symmetry and multivalency, PVX has been explored as a vaccine carrier for infectious diseases as well as cancer. ${ }^{22-26}$ The shape and flexibility of PVX makes it a highly efficient platform for enhanced tumor homing and penetration based on the enhanced permeability and retention (EPR) effect and therefore has tremendous potential as a nanoplatform for delivering therapeutics and imaging contrast agents to solid tumors. ${ }^{27,28}$ Studies pertaining to the biological fate of PVX, however, have so far been limited to single intravenous administration and fail to address particle fate upon repeat exposures. 
Here, we evaluate the immune response generated after multiple intravenous injections of PVX particles and its impact on particle clearance using a combination of conventional ELISA-based serum antibody detection and real time imaging of VNP circulation in mice vasculature using a two-photon laser scanning microscopy (2PLSM)-based intravital imaging approach. Our results illustrate induction of a VNP-specific immune response measurable in terms of varying antibody titers, and the effect of these changes on VNP circulation are observable through real time imaging of central nervous system (CNS) vasculature in mice. Overall, our results demonstrate stark differences between single and repeat administrations of PVX and emphasize the need for similar evaluation of other nanoparticle-based platform technologies in order to obtain further insight into the biological outcomes of repeated administrations.

\section{MATERIALS AND METHODS}

PVX Propagation

PVX was propagated in Nicotiana benthamiana plants and purified using previously described procedures. ${ }^{19,27}$ Purified VNPs were stored in $0.1 \mathrm{M}$ potassium phosphate buffer at $\mathrm{pH} 7.0$ and $4{ }^{\circ} \mathrm{C}$. The concentration of the VNPs was determined by UV-vis spectroscopy $\left(\varepsilon_{\mathrm{PVX}}=2.97 \mathrm{~mL} \mathrm{mg}^{-1} \mathrm{~cm}^{-1}\right.$ at $260 \mathrm{~nm}$ ). Tobacco mosaic virus (TMV), a rigid nanorod measuring $300 \times 18 \mathrm{~nm}$, was similarly propagated in Nicotiana benthamiana plants. The concentration of the TMV was determined by UV-vis spectroscopy $\left(\varepsilon_{\mathrm{TMV}}=3.0 \mathrm{~mL} \mathrm{mg}^{-1}\right.$ $\mathrm{cm}^{-1}$ at $260 \mathrm{~nm}$ ). The isolation of either VNP using established procedures yielded approximately $1 \mathrm{mg}$ of virus particle per gram of infected leaf material. ${ }^{33}$

\section{Synthesis of PVX-A647 VNPs}

PVX was covalently modified with AlexaFluor 647 (A647) dye using $N$ hydroxysuccinimide-activated esters targeting surface-exposed lysine residues on the viral coat proteins. PVX was incubated overnight at room temperature with a 5000 molar excess of NHS-A647 dyes (Life Technologies) in the presence of DMSO (at a final concentration of $10 \%(\mathrm{v} / \mathrm{v}))$. Dye-conjugated PVX was then purified using ultracentrifugation on a $40 \%$ $(\mathrm{w} / \mathrm{v})$ sucrose cushion. UV-vis spectroscopy performed on a Nanodrop instrument was used to determine the A647/PVX ratio based on Beer-Lambert law and the fluorophore and $\mathrm{PVX}$-specific extinction coefficients of $\varepsilon_{\mathrm{A} 647}=270000 \mathrm{M}^{-1} \mathrm{~cm}^{-1}$ at $650 \mathrm{~nm}$ and $\varepsilon_{\mathrm{PVX}}=$ $2.97 \mathrm{~mL} \mathrm{mg}^{-1} \mathrm{~cm}^{-1}$ at $260 \mathrm{~nm}$, respectively.

\section{Transmission Electron Microscopy (TEM)}

TEM analysis was used to ascertain the structural integrity of PVX postmodification and for serum antibody mediated aggregation of PVX and TMV nanoparticles. Diluted samples of PVX-A647 (20 $\left.\mu \mathrm{L}, 0.1 \mathrm{mg} \mathrm{mL}^{-1}\right)$ were negatively stained with $2 \%(\mathrm{w} / \mathrm{v})$ uranyl acetate for 2 min on a carbon-coated copper grid. Samples were analyzed using a FEI Tecnai G2 Spirit transmission electron microscope at $100 \mathrm{kV}$. For serum-mediated aggregation, $2 \mu \mathrm{g}$ of PVX or TMV were incubated with $1 \mu \mathrm{L}$ of naïve mouse sera or PVX-immunized mice sera. TEM samples were then prepared as described above. 


\section{Denaturing Gel Electrophoresis}

SDS-PAGE was used to determine successful conjugation of A647 dye molecules with PVX coat proteins. First, $10 \mu \mathrm{g}$ of denatured protein samples (PVX and PVX-A647) were loaded and run on 4-12\% NuPage gels (Life Technologies) in $1 \times$ MOPS SDS running buffer (Life Technologies). The fluorescence of PVX-A647 was visualized using Maestro fluorescence imager (PerkinElmer) with yellow excitation/emission filters (excitation filter, 575-605; emission filter, 645 long-pass), then protein was visualized under white light after staining with SimplyBlue SafeStain (ThermoFisher Scientific).

\section{Size Exclusion Chromatography (SEC)}

PVX-A647 nanoparticle preparations were analyzed by SEC/FPLC using a Superose6 column on the ÄKTA Explorer chromatography system (GE Healthcare). Samples (100 $\mu \mathrm{L}$ of $1 \mathrm{mg} \mathrm{mL}^{-1}$ ) were analyzed at a flow rate of $0.5 \mathrm{~mL} \mathrm{~min}^{-1}$, using $0.1 \mathrm{M}$ potassium phosphate buffer ( $\mathrm{pH}$ 7.4).

\section{Determination of Anti-PVX Antibody Levels Following Multiple Administrations of PVX}

C57BL/6-Tg(UBC-GFP)-30Scha/J (Stock \# 004353) mice were obtained from The Jackson Laboratory (Bar Harbor, ME, USA). Animals were housed, bred, and handled in the Case Western Reserve University Animal Resource Center in accordance with approved Institutional Animal Care and Use Committee experimental protocols. PVX-specific serum IgG and IgM antibody titers were determined following repeat administration of PVX-A647. Mice were retro-orbitally injected with $200 \mu \mathrm{g}$ of PVX-A647 on days 0,7 , and 14. Blood was collected through tail bleed in heparin-coated tubes (Greiner Bio-One VACUETTE MiniCollect tubes, Thermo Fisher Scientific, Waltham, MA) $24 \mathrm{~h}$ before each injection and 5 min postinjection. Serum was separated by centrifuging blood samples at $14800 \mathrm{rpm}$ for $10 \mathrm{~min}$ and then stored at $4{ }^{\circ} \mathrm{C}$ until being analyzed. Serum antibody specificity was determined using ELISA analysis. Serum collected prior to the first injection was used as naïve serum for each mouse, and successive bleeds were compared to the naïve serum for changing antibody levels. Next, 96-well Nunc Polysorb Immuno plates (Thermo Fisher Scientific, Waltham, MA) were coated with $1 \mu \mathrm{g}$ of PVX/well in coating buffer $(0.05 \mathrm{M}$ $\mathrm{Na}_{2} \mathrm{CO}_{3}, 0.05 \mathrm{M} \mathrm{NaHCO}_{3}, 0.015 \mathrm{M} \mathrm{NaN}_{3}$ in $\mathrm{dH}_{2} \mathrm{O}, \mathrm{pH}$ 9.6) and incubated overnight at $4{ }^{\circ} \mathrm{C}$. Following coating, wells were blocked using $200 \mathrm{~mL} /$ well blocking buffer $(2.5 \%$ (w/v) milk, $25 \%$ (v/v) FBS in $1 \times \mathrm{PBS}, \mathrm{pH} 7.4$ ) at $37^{\circ} \mathrm{C}$ for $1 \mathrm{~h}$. After blocking, $1: 100 \mu \mathrm{L}$ uf sera (in blocking buffer) was added to the wells and incubated at $37^{\circ} \mathrm{C}$ for $2 \mathrm{~h}$. After serum incubation, $100 \mu \mathrm{L}$ of alkaline phosphatase-labeled goat antimouse IgG (Life Technologies, Grand Island, NY) or goat antimouse IgM (Life Technologies, Grand Island, NY) at 1:3000 (in blocking buffer) was added to each well and incubated at $37^{\circ} \mathrm{C}$ for $1 \mathrm{~h}$. In between each step, plates were washed four times with washing buffer $(0.1 \%$ (v/v) Tween-20 in PBS, 200 $\mu \mathrm{L} /$ well). Wells were developed by adding $100 \mu \mathrm{L}$ of one-step PNPP substrate (Thermo Fisher Scientific, Waltham, MA) for $10 \mathrm{~min}$ at $4{ }^{\circ} \mathrm{C}$. Reaction was stopped using $100 \mu \mathrm{L}$ of 2 $\mathrm{M} \mathrm{NaOH}$. Absorbance was read at $405 \mathrm{~nm}$ using a Tecan Infinity 200 microplate reader. 


\section{P-LSM and Imaging Analysis of Multiple Administrations of PVX in Vivo}

In a parallel study, an independent cohort of C57BL/6-Tg(UBC-GFP)30Scha/J mice was used to image CNS vasculature following weekly PVX-A647 administrations. These mice were maintained on a Teklad $2018 \mathrm{~S}$ alfalfa free diet (Harlan Lab, USA) starting 2 weeks before imaging to reduce autofluorescence. Animals were given open cranial window preparations as previously described. ${ }^{29,30}$ Following a two-week resting period post window implantation, mice were anesthetized with nebulized isofluorane (3\% induction, $2 \%$ maintenance) in 1:1 $\mathrm{O}_{2} /$ air and placed in a stereotactic holder. Mice were then placed in a custom environmental chamber and maintained at a constant animal temperature of $37^{\circ} \mathrm{C}$ via both heating pads and other environmental controls. Temperature, anesthetic depth, and breathing rate (approximately 40-80 breaths per minute) were monitored to ensure animal health and comfort. A Leica SP5 confocal microscope equipped with a 20× water immersion lens (Leica HCX-APO-L, N.A. 1.0) and a tunable 16W Ti/Sapphire IR laser tuned to 800 $\mathrm{nm}$ (Chameleon Coherent, Inc.) was used for intravital 2P-LSM imaging. Four-dimensional $X Y Z T$ images with an $X Y$ dimension of $760 \times 760 \mu \mathrm{m}$ and $Z$ dimension of $100-130 \mu \mathrm{m}$ (collected in steps of $5 \mu \mathrm{m} Z$ intervals) were obtained at $20 \mathrm{~s}$ intervals. Each mouse was administered $200 \mu \mathrm{g}$ of PVX-A647 $\left(\sim 3.4 \times 10^{12}\right.$ particles $)$ on days 0,7 , and 14 . All injections were administered retroorbitally concurrently with imaging. Intravital imaging was carried out for $10 \mathrm{~min}$ preinjection and for $10 \mathrm{~min}$ postinjection on each day. The data sets were analyzed using Imaris (BitPlane, Inc.) to generate images and to determine clearance. Mean fluorescence intensity (MFI) was calculated from the signal in all $X Y Z$ dimensions throughout the imaging time course. Clearance was then graphed by normalizing subsequent time points to the MFI of the initial injection $(t=0)$.

\section{RESULTS}

\section{Synthesis and Characterization of Fluorescently Labeled PVX Filaments}

PVX was produced through farming in $N$. benthamiana plants using previously established protocols. ${ }^{19,27}$ PVX filaments were chemically modified with fluorescent dyes AlexaFluor 647 (A647) at solvent-exposed lysine side chains using $N$-hydroxysuccinimide (NHS) chemistry (Figure 1a). TEM analysis confirmed the structural integrity of PVX filaments following dye conjugation and purification steps (Figure 1b). UV-vis spectroscopy indicated conjugation of $\sim 450 \mathrm{~A} 647$ molecules per PVX, i.e., a fluorophore at every third unit of the 1270 identical coat protein copies (Figure 1c). Stability of PVX-A647 over the course of multiple administrations was confirmed using weekly UV-vis and TEM analysis.

To further confirm covalent attachment of A647 to the PVX coat proteins, samples were characterized by denaturing gel electrophoresis. Visualization of fluorescence protein bands confirmed the conjugation of A647 molecules to the $\sim 28 \mathrm{kDa}$ PVX coat protein (Figure 1d). FPLC elution profiles using a Superose6 column and Akta Purifier were consistent with the TEM data, indicating PVX remained structurally intact. Further, the FPLC size exclusion chromatography showed that PVX and A647 (measured at $650 \mathrm{~nm}$ ) have matched elution profiles, therefore indicating that A647-PVX coelutes and that free A748 is not detectable (Figure 1e). Zeta potential analysis of the dye-tagged particles gave positive zeta potential 
values of $+16.8 \pm 8 \mathrm{mV}$ for PVX-A647. The stability of PVX-A647 over the course of multiple administrations was confirmed using weekly UV-vis and TEM analysis.

\section{Carrier-Specific Antibody Response to Multiple Administrations of PVX-A647}

C57BL/6-Tg(UBC-GFP) mice ( $n=3)$ were intravenously injected with PVX-A647 nanoparticles on days 0,7 , and 14 (injections I-1, I-2, and I-3, respectively; Figure 2a). This injection schedule mirrors the clinical setting where, for example, patients receive weekly chemotherapy. ${ }^{31-33}$ Also, longitudinal imaging protocols to assess treatment success may be carried out at similar time frames. We sought to determine PVX-specific serum IgM and IgG antibody levels resulting from the repeat administrations of PVX. Blood was drawn from naïve mice $(n=3)$ via tail-bleed a day prior to first injection I-1 (bleed B1) and prior to injections I-2 and I-3 (B3 and B5, respectively). Similarly, to determine antibody levels following injections, blood was also collected 5 min following each injection I-1, I-2, and I-3 (bleeds B2, B4, and B6, respectively).

ELISA measurements for both IgM and IgG were carried out using PVX-coated plates, thereby detecting only PVX-specific antibody levels in mice serum. IgM and IgG levels following PVX administrations were compared to the preinjection (B1) levels for all mice. While normal levels of natural circulating IgM antibodies have been implicated in neutralization of viruses and viral like particles (VLPs), ${ }^{15,34,35}$ serum IgM levels in the preinjection samples from naïve mice suggested the absence of any preformed PVX-specific IgM antibodies (Figure 2b-B1). Serum collected 5 min following the first PVX injection (I-1) also remained negative for PVX-specific IgM antibodies (Figure 2b-B2). Six days post PVX administration, significant levels of PVX-specific IgM antibodies were detected in mice serum (Figure 2b-B3), suggesting stimulation of an IgM mediated response against PVX. All mice were then injected with a second dose of PVX-A647 particles on day 7 (I-2). As with the first injection, blood was drawn 5 min after the second injection and mice serum analyzed for PVX-reactive antibodies. Interestingly, serum IgM levels dropped significantly after the second dose of PVX particles (Figure 2b-B4). It is likely that the influx of a high dose of antigen $\left(\sim 3.4 \times 10^{12} \mathrm{PVX}\right.$ particles $)$ in the immunized mice rapidly led to formation of PVX-IgM immune complexes, thereby significantly lowering circulating IgM levels (B4). A similar decrease in IgM levels following antigen administration has been previously reported for heat killed bacterial antigens ${ }^{36}$ as well as PEGylated liposomes. ${ }^{37,38}$ (This, in fact, was visualized by intravital microscopy, see below.)

Nevertheless, this decrease of IgM level with PVX administration was only observed upon first repeat admin- istration (I-2), and not at subsequent administrations. Further, after the first booster of PVX, we observed a 2-fold increase in PVX-specific IgM (Figure 2b-B5). It is possible that while PVX-IgM immune complexes still formed, higher titers of PVXspecific IgM preceding the third PVX injection were able to neutralize PVX antigens without significantly affecting the overall circulating IgM levels.

PVX-specific IgG levels were also monitored alongside IgM levels. Antigen-specific IgG is produced via class switching which occurs when naïve $\mathrm{B}$ cells that produce antigen-specific IgM antibodies are activated and proliferate to produce IgG antibodies with conserved variable regions and hence retain specificity to the antigen. ${ }^{39}$ As expected, mice serum prior 
to PVX administration did not harbor any preexisting PVX-reactive IgG (Figure 2c-B1), and significant IgG levels were only detected 2 weeks after the first administration of PVX (Figure 2c-B5), a time lapse that is typical for the development of antigen-specific IgG response. ${ }^{39,40} \mathrm{IgG}$ levels varied between the three immunized mice; however, the one mouse with low levels of $\mathrm{IgG}$ also showed much lower IgM levels, indicating a weaker overall immune response. IgG levels dropped significantly immediately following the third PVX injection (Figure 2c-B6), suggesting the formation of PVX-IgG immune complexes and consequently lowering of circulating free IgG antibodies detectable by ELISA. Participation of IgG antibodies in the formation of immune complexes with PVX could also contribute to a relatively smaller change in circulating IgM levels following the third injection (Figure 2bB6), indicating dominance of IgG-to-PVX interactions over IgM-to-PVX interactions. It has been established that as a result of class switching and affinity maturation, IgG outcompetes IgM for protein antigens, ${ }^{41-43}$ which also supports the preferential IgG-PVX interactions observed here.

To demonstrate the ability of PVX-specific antibodies ( $\operatorname{IgG}$ and $\operatorname{IgM})$ to form immune complexes in vitro, we incubated PVX with naïve mice sera (B1) or immunized mice sera (B6) and imaged these samples with TEM (Figure 3). PVX filaments showed moderate aggregation with naïve sera, which is likely due to nonspecific interactions with a large number of serum proteins, including nonreactive antibodies naturally present in the mice sera (Figure $3 \mathrm{~b}$ and inset). Once incubated with immunized sera, significant clustering and formation of large bundles comprised of PVX filaments was observed (Figure $3 \mathrm{c}$ and inset). Similar clustering of influenza virus in the presence of IgM has been shown in vitro. ${ }^{34}$

To demonstrate that the aggregation of PVX in vitro was mediated by carrier-specific antibodies, we incubated tobacco mosaic virus (TMV) with sera from naïve (B1) and PVX immunized (B6). TMV is a well-established rigid rod-shaped plant viral nanoparticle e4,45 $^{4}$ with comparable size and molecular weight to PVX. Upon incubation, neither the sera from naïve mice nor PVX immunized mice led to TMV aggregation (Figure 3d-f). Together, these results clearly show high specificity of immunized sera for PVX nanoparticles.

\section{Intravital Imaging for in Vivo Monitoring of VNPs in Circulation}

Intravital imaging using 2P-LSM enables real time monitoring of vasculature and circulation and is an excellent tool for in situ quantitative pharmacokinetics analysis of fluorescently tagged nanoparticles. ${ }^{46-50}$ It offers high sensitivity and temporal resolution, while facilitating multiple imaging analyses on individual mice. Together, these advantages make intravital imaging an attractive technique for studying the fates of nanoparticles in vivo. Toward this end, we subjected a cohort of C57BL/6-Tg(UBC-GFP) mice $(n=3)$ to a PVX injection schedule similar to the one used above. These mice express green fluorescent protein (GFP) in all tissues under the control of the human ubiquitin $\mathrm{C}$ promoter ${ }^{51}$ and therefore enable visualization of vasculature clearly via absence of a fluorescence signal as compared to the surrounding tissue (Supporting Information S1).

The CNS vasculature was imaged through the cranial windows using a stereotactic holder to stabilize the cranium (Figure 4a). Mice were injected at seven-day intervals with PVX-A647 particles (Figure 4b), and four-dimensional (XYZT) images were captured for $10 \mathrm{~min}$ prior 
to VNP injections and up to 10 min continuously postinjection (Supporting Information S2). We chose open window craniotomies because it gives the opportunity for multiple imaging sessions over several weeks. ${ }^{52,53}$ Allowing animals to rest for 2 weeks postcraniotomy ensured that no procedural induced inflammation would affect immune-based VNP clearance. Additionally, we chose this platform because of the image stability it provides over multiple acquisitions and because it provided access for retro-orbital injection permitting us to image immediately following VNP injection (Figure 4c).

The dye-tagged VNPs produced bright fluorescence within the vasculature. At the time of first injection, the circulating PVX-A647 particles showed uniform brightness throughout the vessels (Figure 4c, I-1). The fluorescence signal intensity then degraded rapidly within the first 10 min (Supporting Information Figure S2), and the clearance rate did not change significantly beyond the first $10 \mathrm{~min}$ (data not shown). These intravital results mirror our previous in vivo pharmacokinetics studies, which have established a $\sim 12$ min circulation half-life of PVX particles ${ }^{19,27}$ — with the largest decrease in percentage of circulating VNPs observed within the first $5 \mathrm{~min}$, after which the particle concentration gradually falls to undetectable levels. Therefore, to compare any visibly detectable changes in nanoparticle flow upon repeat administration, we imaged the mice vasculature for 10 min following the injections. Additionally, the $10 \mathrm{~min}$ imaging period also allowed us to avoid imaging drift artifacts which can cause short breaks in acquisition (as evidenced at $t=10 \mathrm{~min}$, Figure $4 \mathrm{~d}$ : I-1). The uniform brightness of particles within the vasculature is consistent with similarly imaged nanoparticle formulations in several other studies following single injections. ${ }^{50,54}$

In contrast to I-1, at the second injection on day 7, a fluorescence signal from PVX-A647 appeared in the form of clusters throughout the vasculature immediately following the injection (Figure 4c, I-2). The apparent aggregation of PVX particles within the vasculature correlates well with ELISA data showing the presence of PVX-reactive IgM antibodies in the circulation prior to injection and the significant drop in IgM levels 5 min following the injection (Figure 2b-B3/B4). Moreover, formation of large aggregates of PVX in vitro upon incubation with sera from immunized mice (Figure 3c) also indicated that the clusters observed in intravital imaging resulted from the rapidly forming PVX-IgM immune complexes. ${ }^{55-57}$ The formation of such large PVX-IgM complexes resulted in removal of circulating serum IgM antibodies as indicated by lower levels detected through ELISA measurements.

Interestingly though, the third dose of PVX-A647 at day 14 (I-3) resulted in fewer clusters and a partial restoration of uniform fluorescent intensity as observed at first injection (Figure 4c, I-3). ELISA results showed minimal variation between the preinjection and postinjection PVX-reactive IgM antibody levels (for injection 3), suggesting that a significantly smaller fraction of IgM antibodies formed immune complexes with circulating PVX particles. In contrast, PVX-reactive IgG levels dropped significantly following the third injection, suggesting the participation of IgG molecules in PVX neutralization. It is likely that the monomeric IgG forms smaller and fewer aggregates than the pentameric IgM molecule and therefore engaged a relatively smaller fraction of circulating PVX in immune complexes. Taken together, these results highlight the role of PVX-specific antibodies in determining the fate of viral nanoparticles immediately following their intravascular introduction. 
Intravital imaging has been previously employed to quantify pharmacokinetics and tissue accumulation of nanoparticles with longer circulating half-lives ${ }^{50,54}$ Using image analysis, we measured the changes in mean fluorescence intensity (MFI) of circulating PVX-A647 nanoparticles over time (Figure 4d). The MFI values were normalized with respect to the fluorescence intensities immediately after injections, indicating a steady decline in values from a maximum intensity (Figure 4d). While the aggregation patterns observed following successive injections were consistent between mice, the average MFI values (average of three mice) did not show significant differences between I-1, I-2, and I-3. This suggests that current settings employed and the small cohort of mice used may not be optimum for MFI analysis. Nonetheless, these results do highlight the nonuniformity of fluorescence signal following repeat administration caused by immune complex formations, phenomena that were largely unaddressed in prior intravital nanoparticle studies. Our results also underline the importance of intravital imaging in monitoring repeat administration of nanoparticles, as mere quantitative analysis of fluorescence signals without imaging may not be able to highlight significant changes in particle clearance so apparent in the vasculature.

\section{DISCUSSION}

Clinical use of nanoparticle-based formulations for delivery of therapeutics or contrast agents requires repeated doses over the course of treatment or monitoring for diagnostic imaging. While the rapid clearance of a large fraction of injected nanocarriers by RES is itself an obstacle in attaining desirable doses at targeted sites, the induction of a carrierspecific immune response upon repeat administrations could further diminish the efficiency of such an approach. Immune recognition of the nanocarrier could enhance its clearance and off-target accumulation, resulting in altered pharmacokinetics, and could potentially cause unwanted systemic toxicities. These effects are likely to be more pronounced for nanoparticles of biological origins, such as protein cage nanoparticles and viral nanoparticles of plant and bacterial origin, and demand careful preclinical evaluation.

Toward this end, we evaluated the immune response triggered by multiple administrations of plant based PVX VNPs in mice. Humoral immunity, particularly secreted neutralizing antibodies, is primarily responsible for clearance of viruses from circulation. ${ }^{58,59} \mathrm{In}$ fact, a carrier specific antibody response has been identified as mediator of clearance of viral vectors, ${ }^{18,60}$ protein cage nanoparticles, ${ }^{61}$ and PEGylated liposomes. ${ }^{38}$ Based on the potential to effectively display B-cell epitopes and generate antibody responses, PVX and other VNPs have been used as vaccine platforms leading to epitope- and carrier-specific antibodies. ${ }^{62}$ Earlier studies have also highlighted the affinity of PVX to B-cell-rich regions in secondary lymphoid organs. ${ }^{28}$ On the basis of these observations, we predicted induction of an antibody-mediated immune response upon repeat administration. Our results illustrate that initially anti-PVX IgM antibodies primarily dictate the clearance of PVX particles. This is in agreement with established literature that both naturally occurring IgM antibodies in naïve sera as well as stimulated immune IgM play a key role in protection against several viruses. ${ }^{56}$ The absence of in vitro aggregation of PVX when incubated with naïve sera (Figure 3) as well as the uniformity of fluorescent signals during imaging (Figure 4c, I-1) indicate that PVX in circulation is not affected by pre-existing nonspecific IgM in naïve sera. Furthermore, the immunoglobulin responses elicited by repeated administrations of 
PVX are specific for PVX epitopes as evidenced by their failure to cause aggregation of TMV in vitro. Neither naïve nor PVX-immunized sera caused aggregation of TMV, although our earlier work indicated that the protein corona formed on TMV upon exposure to plasma is dominated by complement, plasminogen, and IgM. ${ }^{63}$ Nevertheless, the titers are apparently too low to affect the circulation of the carriers at first administration and also correlates with a low level of VNP-specific circulating antibodies detected by ELISA in the naïve mice (Figure 3).

Six days following the first injection, a significant increase in anti-PVX IgM was noted in two out of three mice with a subsequent significant drop in anti-PVX IgM levels as observed by ELISA following the second injection. These data, taken together with the appearance of large clusters in the vasculature during imaging, suggest the formation of IgM-PVX immune complexes. By virtue of its multivalency, IgM molecules are known to form large immune complexes with antigens and promote hepatic clearance of the antigen. ${ }^{56}$ Similar drops in IgM levels have been reported following administration of heat killed bacterial antigen ${ }^{36}$ and repeat administration of PEGylated liposomes containing doxorubicin. ${ }^{38}$ These fluctuating levels of IgM have been correlated with the accelerated clearance of such PEGylated liposomes. ${ }^{37,64}$ It should be noted that C57BL/6-Tg(UBC-GFP) mice allow for visualization of GFP-expressing circulating cells which include phagocytic neutrophils and monocytes. We observed no significant colocalization between the bright clusters of fluorescently tagged PVX nanoparticles and $\mathrm{GFP}^{+}$cells in circulation at the second injection (Supporting Information S3), further supporting our hypothesis of circulating IgM as the primary player in formation of these clusters.

A key step in development of humoral immune response is class switching of antibody isotypes from IgM to IgG. We observed this switch inherent with increasing levels of antiPVX IgG starting 2 weeks after the first injection. After the third injection, the VNP-specific IgG levels show a significant drop, whereas the IgM levels are relatively constant (Figure 3). This switch in antibody class is reflected in vivo as fewer large clusters of fluorescent PVX are observed by intravital imaging (Figure 4). These results point to the involvement of the IgG monomers forming smaller immune complexes with PVX nanoparticles than their pentameric IgM counterparts.

Thus, it is clear from the above results that multiple administrations of PVX lead to an immune response that could significantly influence circulation half-life, clearance, and sequestration of VNPs. Although it is accepted that the immunogenic properties of nanoparticles, protein-based carriers, ${ }^{65,66}$ as well as plant viruses ${ }^{67}$ can be attenuated by stealth polymer coatings such as polyethylene glycol (PEG), ${ }^{66,68-71}$ extensive preclinical evaluation upon multiple administration of these nanoparticles is important as it could advance findings that may be useful for designing more efficient nanocarriers for clinical treatments. We have established intravital microscopy as a valuable tool to aid the investigation of the in vivo fates of (viral) nanoparticles after repeated administration, which allows for a real-time read-out of VNP fate. In order to utilize intravital microscopy for quantitative pharmacokinetic analysis of fluorescent nanocarriers, additional image analysis methods may be required to decouple the signal intensity of freely floating and immune complex-bound fractions of the nanocarriers. Additionally, in future studies, nanoparticle 
formulations could be evaluated as a function of stealth or camouflage coating to determine the most effective formulation of cargo-loaded carriers that enables repeat administration for medical applications. At the same time, we envision that the techniques and findings presented here will aid with the development of dosing schedules for vaccine development or therapeutic cargo delivery.

\section{CONCLUSIONS}

Viral nanoparticles such as PVX offer a highly organized proteinaceous structure and multivalency. With the opportunity for precise genetic and chemical engineering, VNPs are therefore ideally suited for delivering large payloads of therapeutic molecules and imaging contrast agents. These very structural features could also result in immunological recognition and a subsequent rapid clearance of VNPs from circulation, altering their overall efficiency. These shortcomings could have a major impact upon repeat administration over the course of therapy or longitudinal disease monitoring. Therefore, studies pertinent to multiple administrations of VNPs are critical for successful advancement of this promising platform technology.

Using conventional methods such as ELISA and TEM analysis, we have demonstrated the generation of a VNP-specific immune response, mediated initially by IgM and later by $\operatorname{IgG}$, in response to weekly intravenous administrations of PVX. The antibody response resulted in the formation of immune complexes that led to the aggregation of PVX, which could be visualized in real time with $2 \mathrm{P}$-LSM based intravital imaging. Thus, we demonstrated that repeat administration of plant-based virus nanoparticles triggered a specific antibody response that could potentially alter the in vivo behavior and effectiveness of these VNP nanoformulations for drug delivery and imaging applications. The contrasting nature of particle behavior in circulation following single and multiple injections underlines the need for similar evaluation of a broader range of nanoparticle-based platform technologies for clinical translation.

\section{Supplementary Material}

Refer to Web version on PubMed Central for supplementary material.

\section{Acknowledgments}

This work was partially funded by NCI F31CA196265 (R.D.D.), R01CA154656 (A.Y.H.), R21CA181875 (A.Y.H.), a Hyundai Hope Grant (A.Y.H.), as well as a grant from NIBIB R03EB020602 (N.F.S.) and a grant from the American Cancer Society 128319-RSG-15-144-01-CDD (N.F.S.).

\section{REFERENCES}

1. Davis ME, Chen ZG, Shin DM. Nanoparticle therapeutics: an emerging treatment modality for cancer. Nat. Rev. Drug Discovery. 2008; 7:771-782. [PubMed: 18758474]

2. Wang AZ, Langer R, Farokhzad OC. Nanoparticle delivery of cancer drugs. Annu. Rev. Med. 2012; 63:185-198. [PubMed: 21888516]

3. Petros RA, DeSimone JM. Strategies in the design of nanoparticles for therapeutic applications. Nat. Rev. Drug Discovery. 2010; 9:615-627. [PubMed: 20616808] 
4. Blanco E, Shen H, Ferrari M. Principles of nanoparticle design for overcoming biological barriers to drug delivery. Nat. Biotechnol. 2015; 33:941-951. [PubMed: 26348965]

5. Wolfram J, Zhu M, Yang Y, Shen J, Gentile E, Paolino D, Fresta M, Nie G, Chen C, Shen H, Ferrari M, Zhao Y. Safety of Nanoparticles in Medicine. Curr. Drug Targets. 2014; 16:1671.

6. Mitragotri S, Anderson DG, Chen X, Chow EK, Ho D, Kabanov AV, Karp JM, Kataoka K, Mirkin CA, Petrosko SH, Shi J, Stevens MM, Sun S, Teoh S, Venkatraman SS, Xia Y, Wang S, Gu Z, Xu C. Accelerating the Translation of Nanomaterials in Biomedicine. ACS Nano. 2015; 9:6644-6654. [PubMed: 26115196]

7. Aggarwal P, Hall JB, McLeland CB, Dobrovolskaia MA, McNeil SE. Nanoparticle interaction with plasma proteins as it relates to particle biodistribution, biocompatibility and therapeutic efficacy. Adv. Drug Delivery Rev. 2009; 61:428-437.

8. Gao H, He Q. The interaction of nanoparticles with plasma proteins and the consequent influence on nanoparticles behavior. Expert Opin. Drug Delivery. 2014; 11:409-420.

9. Yildiz I, Shukla S, Steinmetz NF. Applications of viral nanoparticles in medicine. Curr. Opin. Biotechnol. 2011; 22:901-908. [PubMed: 21592772]

10. Lewis JD, Destito G, Zijlstra A, Gonzalez MJ, Quigley JP, Manchester M, Stuhlmann H. Viral nanoparticles as tools for intravital vascular imaging. Nat. Med. 2006; 12:354-360. [PubMed: 16501571]

11. Steinmetz NF. Viral nanoparticles as platforms for next-generation therapeutics and imaging devices. Nanomedicine. 2010; 6:634-641. [PubMed: 20433947]

12. Pokorski JK, Steinmetz NF. The art of engineering viral nanoparticles. Mol. Pharmaceutics. 2011; 8:29-43.

13. Scheerlinck JP, Greenwood DL. Virus-sized vaccine delivery systems. Drug Discovery Today. 2008; 13:882-887. [PubMed: 18656548]

14. Jobsri J, Allen A, Rajagopal D, Shipton M, Kanyuka K, Lomonossoff GP, Ottensmeier C, Diebold SS, Stevenson FK, Savelyeva N. Plant virus particles carrying tumour antigen activate TLR7 and Induce high levels of protective antibody. PLoS One. 2015; 10:e0118096. [PubMed: 25692288]

15. Link A, Zabel F, Schnetzler Y, Titz A, Brombacher F, Bachmann MF. Innate immunity mediates follicular transport of particulate but not soluble protein antigen. J. Immunol. 2012; 188:37243733. [PubMed: 22427639]

16. Plummer EM, Manchester M. Viral nanoparticles and virus-like particles: platforms for contemporary vaccine design. Wiley Interdiscip Rev. Nanomed Nanobiotechnol. 2011; 3:174. [PubMed: 20872839]

17. Lizotte PH, Wen AM, Sheen MR, Fields J, Rojanasopondist P, Steinmetz NF, Fiering S. In situ vaccination with cowpea mosaic virus nanoparticles suppresses metastatic cancer. Nat. Nanotechnol. 2015; 11:295. [PubMed: 26689376]

18. Kotterman MA, Yin L, Strazzeri JM, Flannery JG, Merigan WH, Schaffer DV. Antibody neutralization poses a barrier to intravitreal adeno-associated viral vector gene delivery to nonhuman primates. Gene Ther. 2015; 22:116-126. [PubMed: 25503696]

19. Lee KL, Shukla S, Wu M, Ayat NR, El Sanadi CE, Wen AM, Edelbrock JF, Pokorski JK, Commandeur U, Dubyak GR, Steinmetz NF. Stealth filaments: Polymer chain length and conformation affect the in vivo fate of PEGylated potato virus X. Acta Biomater. 2015; 19:166179. [PubMed: 25769228]

20. Bruckman MA, Randolph LN, Gulati NM, Stewart PL, Steinmetz NF. Silica-coated Gd(DOTA)loaded protein nano-particles enable magnetic resonance imaging of macrophages. J. Mater. Chem. B. 2015; 3:7503-7510.

21. Pitek AS, Jameson SA, Veliz FA, Shukla S, Steinmetz NF. Serum albumin 'camouflage' of plant virus based nanoparticles prevents their antibody recognition and enhances pharmacokinetics. Biomaterials. 2016; 89:89-97. [PubMed: 26950168]

22. Massa S, Simeone P, Muller A, Benvenuto E, Venuti A, Franconi R. Antitumor activity of DNA vaccines based on the human papillomavirus-16 E7 protein genetically fused to a plant virus coat protein. Hum. Gene Ther. 2008; 19:354-364. [PubMed: 18439124] 
23. Plchova H, Moravec T, Hoffmeisterova H, Folwarczna J, Cerovska N. Expression of Human papillomavirus 16 E7ggg oncoprotein on $\mathrm{N}$ - and C-terminus of Potato virus X coat protein in bacterial and plant cells. Protein Expression Purif. 2011; 77:146-152.

24. Lico C, Mancini C, Italiani P, Betti C, Boraschi D, Benvenuto E, Baschieri S. Plant-produced potato virus $X$ chimeric particles displaying an influenza virus-derived peptide activate specific CD8+ T cells in mice. Vaccine. 2009; 27:5069-5076. [PubMed: 19563889]

25. Uhde-Holzem K, Schlosser V, Viazov S, Fischer R, Commandeur U. Immunogenic properties of chimeric potato virus $\mathrm{X}$ particles displaying the hepatitis $\mathrm{C}$ virus hypervariable region I peptide R9. J. Virol. Methods. 2010; 166:12-20. [PubMed: 20138085]

26. Shukla S, Wen AM, Commandeur U, Steinmetz NF. Presentation of HER2 epitopes using a filamentous plant virus-based vaccination platform. J. Mater. Chem. B. 2014; 2:6249-6258.

27. Shukla S, Ablack AL, Wen AM, Lee KL, Lewis JD, Steinmetz NF. Increased tumor homing and tissue penetration of the filamentous plant viral nanoparticle Potato virus X. Mol. Pharmaceutics. 2013; 10:33-42.

28. Shukla S, Wen AM, Ayat NR, Commandeur U, Gopalkrishnan R, Broome AM, Lozada KW, Keri RA, Steinmetz NF. Biodistribution and clearance of a filamentous plant virus in healthy and tumor-bearing mice. Nanomedicine (London, U. K.). 2014; 9:221-235.

29. Barkauskas DS, Evans TA, Myers J, Petrosiute A, Silver J, Huang AY. Extravascular CX3CR1+ cells extend intravascular dendritic processes into intact central nervous system vessel lumen. Microsc. Microanal. 2013; 19:778-790. [PubMed: 23642852]

30. Dorand RD, Barkauskas DS, Evans TA, Petrosiute A, Huang AY. Comparison of intravital thinned skull and cranial window approaches to study CNS immunobiology in the mouse cortex. Intravital. 2014; 3:e29728. [PubMed: 25568834]

31. Bae WK, Hwang JE, Shim HJ, Cho SH, Lee KH, Han HS, Song EK, Yun HJ, Cho IS, Lee JK, Lim SC, Chung WK, Chung IJ. Multicenter phase II study of weekly docetaxel, cisplatin, and S-1 (TPS) induction chemotherapy for locally advanced squamous cell cancer of the head and neck. BMC Cancer. 2013; 13:102. [PubMed: 23497365]

32. Masci G, Gandini C, Zuradelli M, Losurdo A, Torrisi R, Rota S, Gullo G, Velutti L, Giordano L, Santoro A. Weekly non-pegylated liposomal doxorubicin chemotherapy in heavily pre-treated patients with metastatic breast cancer. Anticancer Research. 2013; 33:4603-4609. [PubMed: 24123037]

33. Mori K, Kamiyama Y, Kasai H, Kodama T. A phase II study of first-line chemotherapy with weekly carboplatin plus gemcitabine in advanced non-small cell lung cancer. Chemotherapy. 2012; 58:78-83. [PubMed: 22377616]

34. Jayasekera JP, Moseman EA, Carroll MC. Natural antibody and complement mediate neutralization of influenza virus in the absence of prior immunity. J. Virol. 2007; 81:3487-3494. [PubMed: 17202212]

35. Ochsenbein AF, Fehr T, Lutz C, Suter M, Brombacher F, Hengartner H, Zinkernagel RM. Control of early viral and bacterial distribution and disease by natural antibodies. Science. 1999; 286:2156-2159. PMID: 10591647. [PubMed: 10591647]

36. Coeurdacier JL, Pepin JF, Fauvel C, Legall P, Bourmaud AF, Romestand B. Alterations in total protein, IgM and specific antibody activity of male and female sea bass (Dicentrarchus labrax L, 1758) sera following injection with killed Vibrio anguillarum. Fish Shellfish Immunol. 1997; 7:151-160.

37. Suzuki T, Ichihara M, Hyodo K, Yamamoto E, Ishida T, Kiwada H, Kikuchi H, Ishihara H. Influence of dose and animal species on accelerated blood clearance of PEGylated liposomal doxorubicin. Int. J. Pharm. 2014; 476:205-212. [PubMed: 25280884]

38. Mima Y, Hashimoto Y, Shimizu T, Kiwada H, Ishida T. Anti-PEG IgM Is a Major Contributor to the Accelerated Blood Clearance of Polyethylene Glycol-Conjugated Protein. Mol. Pharmaceutics. $2015 ; 12: 2429-2435$.

39. Xu Z, Zan H, Pone EJ, Mai T, Casali P. Immunoglobulin class-switch DNA recombination: induction, targeting and beyond. Nat. Rev. Immunol. 2012; 12:517-531. [PubMed: 22728528]

40. Vidarsson G, Dekkers G, Rispens T. IgG subclasses and allotypes: from structure to effector functions. Front. Immunol. 2014; 5:520. [PubMed: 25368619] 
41. Makela O, Ruoslahti E, Seppala IJ. Affinity of IgM and IgG antibodies. Immunochemistry. 1970; 7:917-932. [PubMed: 4992788]

42. Honjo T, Kinoshita K, Muramatsu M. Molecular mechanism of class switch recombination: linkage with somatic hypermutation. Annu. Rev. Immunol. 2002; 20:165-196. [PubMed: 11861601]

43. Muthana SM, Xia L, Campbell CT, Zhang Y, Gildersleeve JC. Competition between serum IgG, IgM, and IgA anti-glycan antibodies. PLoS One. 2015; 10:e0119298. [PubMed: 25807519]

44. Shukla S, Eber FJ, Nagarajan AS, DiFranco NA, Schmidt N, Wen AM, Eiben S, Twyman RM, Wege C, Steinmetz NF. The Impact of Aspect Ratio on the Biodistribution and Tumor Homing of Rigid Soft-Matter Nanorods. Adv. Healthcare Mater. 2015; 4:874-882.

45. Bruckman MA, Jiang K, Simpson EJ, Randolph LN, Luyt LG, Yu X, Steinmetz NF. Dual-modal magnetic resonance and fluorescence imaging of atherosclerotic plaques in vivo using VCAM-1 targeted tobacco mosaic virus. Nano Lett. 2014; 14:1551-1558. [PubMed: 24499194]

46. Smith BR, Cheng Z, De A, Rosenberg J, Gambhir SS. Dynamic visualization of RGD-quantum dot binding to tumor neovasculature and extravasation in multiple living mouse models using intravital microscopy. Small. 2010; 6:2222-2229. [PubMed: 20862677]

47. Pittet MJ, Weissleder R. Intravital imaging. Cell. 2011; 147:983-991. [PubMed: 22118457]

48. Leong HS, Steinmetz NF, Ablack A, Destito G, Zijlstra A, Stuhlmann H, Manchester M, Lewis JD. Intravital imaging of embryonic and tumor neovasculature using viral nanoparticles. Nat. Protoc. 2010; 5:1406-1417. [PubMed: 20671724]

49. Ricard C, Debarbieux FC. Six-color intravital two-photon imaging of brain tumors and their dynamic microenvironment. Front. Cell. Neurosci. 2014; 8:57. [PubMed: 24605087]

50. Miller MA, Zheng YR, Gadde S, Pfirschke C, Zope H, Engblom C, Kohler RH, Iwamoto Y, Yang KS, Askevold B, Kolishetti N, Pittet M, Lippard SJ, Farokhzad OC, Weissleder R. Tumourassociated macrophages act as a slow-release reservoir of nano-therapeutic $\mathrm{Pt}(\mathrm{IV})$ pro-drug. Nat. Commun. 2015; 6:8692. [PubMed: 26503691]

51. Schaefer BC, Schaefer ML, Kappler JW, Marrack P, Kedl RM. Observation of antigen-dependent CD8+ T-cell/dendritic cell interactions in vivo. Cell. Immunol. 2001; 214:110-122. [PubMed: 12088410]

52. Mostany R, Portera-Cailliau C. A method for 2-photon imaging of blood flow in the neocortex through a cranial window. J. Visualized Exp. 2008

53. Mostany R, Portera-Cailliau C. A craniotomy surgery procedure for chronic brain imaging. J. Visualized Exp. 2008

54. Huynh AS, Estrella V, Stark VE, Cohen AS, Chen T, Casagni TJ, Josan JS, Lloyd MC, Johnson J, Kim J, Hruby VJ, Vagner J, Morse DL. Tumor Targeting and Pharmacokinetics of a Near-Infrared Fluorescent-Labeled delta-Opioid Receptor Antagonist Agent, Dmt-Tic-Cy5. Mol. Pharmaceutics. 2016; $13: 534$.

55. Skogh T, Blomhoff R, Eskild W, Berg T. Hepatic uptake of circulating IgG immune complexes. Immunology. 1985; 55:585-594. PMCID: PMC1453780. [PubMed: 4018843]

56. Ehrenstein MR, Notley CA. The importance of natural IgM: scavenger, protector and regulator. Nat. Rev. Immunol. 2010; 10:778-786. [PubMed: 20948548]

57. Srivastava AS, Kaido T, Carrier E. Immunological factors that affect the in vivo fate of T7 phage in the mouse. J. Virol. Methods. 2004; 115:99-104. [PubMed: 14656466]

58. Dorner T, Radbruch A. Antibodies and B cell memory in viral immunity. Immunity. 2007; 27:384392. [PubMed: 17892847]

59. Zinkernagel RM, LaMarre A, Ciurea A, Hunziker L, Ochsenbein AF, McCoy KD, Fehr T, Bachmann MF, Kalinke U, Hengartner H. Neutralizing antiviral antibody responses. Adv. Immunol. 2001; 79:1-53. PMID: 11680006. [PubMed: 11680006]

60. Louis Jeune V, Joergensen JA, Hajjar RJ, Weber T. Pre-existing anti-adeno-associated virus antibodies as a challenge in AAV gene therapy. Hum. Gene Ther: Methods. 2013; 24:59-67. [PubMed: 23442094]

61. Kaiser CR, Flenniken ML, Gillitzer E, Harmsen AL, Harmsen AG, Jutila MA, Douglas T, Young MJ. Biodistribution studies of protein cage nanoparticles demonstrate broad tissue distribution and 
rapid clearance in vivo. Int. J. Nanomedicine. 2007; 2:715-733. PMID: 18203438. [PubMed: 18203438]

62. Yusibov V, Rabindran S, Commandeur U, Twyman RM, Fischer R. The potential of plant virus vectors for vaccine production. Drugs R\&amp;D. 2006; 7:203-217.

63. Pitek AS, Wen AM, Shukla S, Steinmetz NF. The Protein Corona of Plant Virus Nanoparticles Influences their Dispersion Properties, Cellular Interactions, and In Vivo Fates. Small. 2016; 12:1758. [PubMed: 26853911]

64. Hashimoto Y, Shimizu T, Abu Lila AS, Ishida T, Kiwada H. Relationship between the concentration of anti-polyethylene glycol (PEG) immunoglobulin M (IgM) and the intensity of the accelerated blood clearance (ABC) phenomenon against PEGylated liposomes in mice. Biol. Pharm. Bull. 2015; 38:417-424. [PubMed: 25757923]

65. Jokerst JV, Lobovkina T, Zare RN, Gambhir SS. Nanoparticle PEGylation for imaging and therapy. Nanomedicine (London, U. K.). 2011; 6:715-728.

66. Veronese FM, Pasut G. PEGylation, successful approach to drug delivery. Drug Discovery Today. 2005; 10:1451-1458. [PubMed: 16243265]

67. Raja KS, Wang Q, Gonzalez MJ, Manchester M, Johnson JE, Finn MG. Hybrid virus-polymer materials. 1. Synthesis and properties of PEG-decorated cowpea mosaic virus. Biomacromolecules. 2003; 3:472-476.

68. Harris JM, Chess RB. Effect of pegylation on pharmaceuticals. Nat. Rev. Drug Discovery. 2003; 2:214-221. [PubMed: 12612647]

69. Jokerst JV, Lobovkina T, Zare RN, Gambhir SS. Nanoparticle PEGylation for imaging and therapy. Nanomedicine (London, U. K.). 2011; 6:715-728.

70. Roberts MJ, Bentley MD, Harris JM. Chemistry for peptide and protein PEGylation. Adv. Drug Delivery Rev. 2002; 54:459-476.

71. Wattendorf U, Merkle HP. PEGylation as a tool for the biomedical engineering of surface modified microparticles. J. Pharm. Sci. 2008; 97:4655. [PubMed: 18306270] 
a)

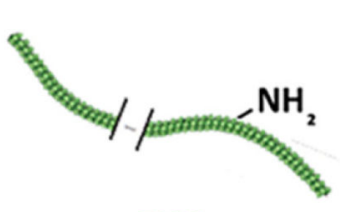

PVX

$515 \times 13 \mathrm{~nm}$

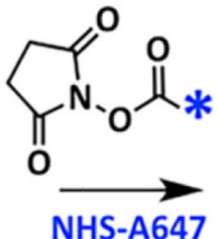

NHS-A647

c)

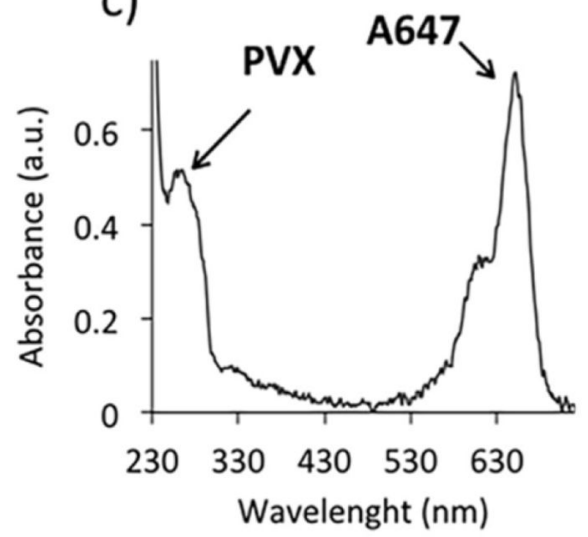

d)

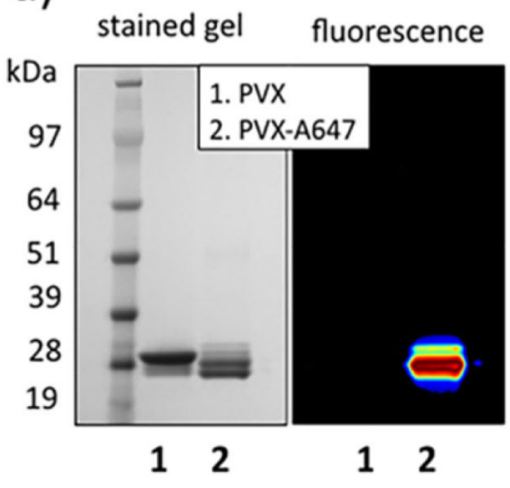

b)

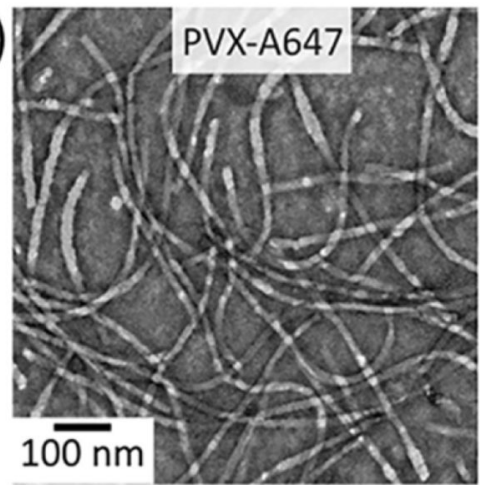

e)
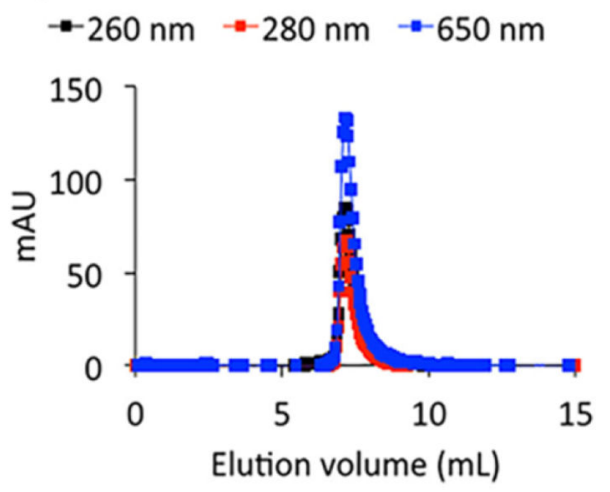

Figure 1.

Synthesis and characterization of PVX-A647 nanoparticles. NHS chemistry was used to conjugate A647 dyes to the solvent exposed Lys side chains of PVX (a). TEM analysis indicated structural integrity of PVX filaments following the chemical conjugation and purifications (b). UV-vis spectroscopy was used to determine the number of A647/PVX (c). Denaturing gel electrophoresis (d) together with coelution of PVX and A647 in FPLC (e) confirmed the specific conjugation of dye molecules with the PVX coat proteins. 
a)

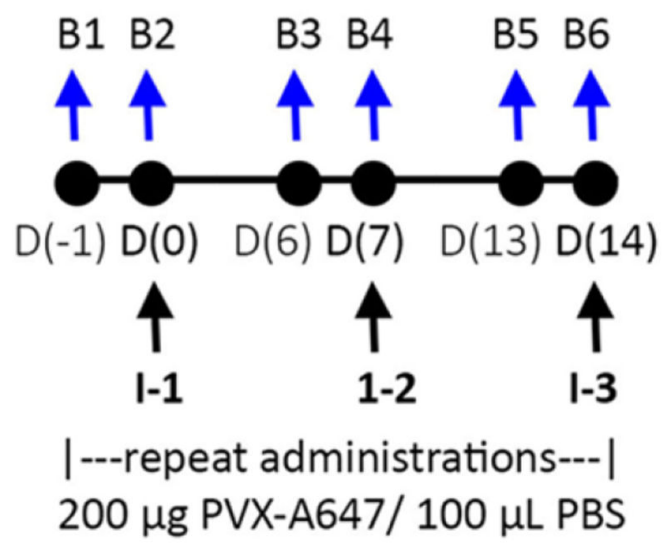

b)

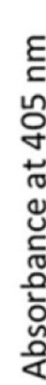

口Mouse \#1 - Mouse \#2 $\square$ Mouse \#3

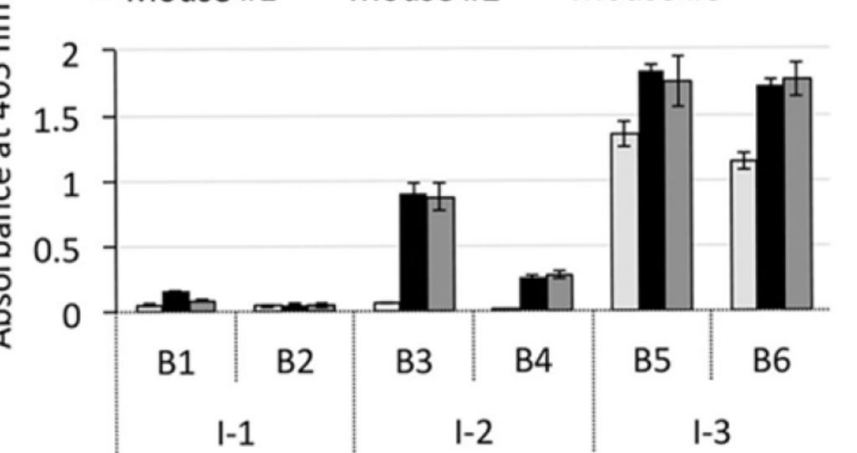

c)

口Mouse \#1 Mouse \#2 $\square$ Mouse \#3

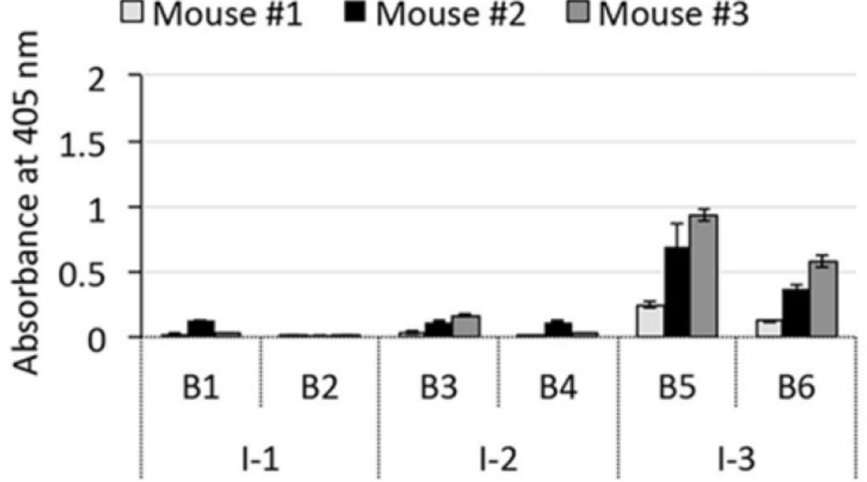

Figure 2.

Repeat administrations of PVX and generation of PVX-specific antibodies. Schematic of multiple administration and bleeding schedules (a). ELISA results showing increasing levels of anti-PVX IgM (b) and IgG responses (c). 

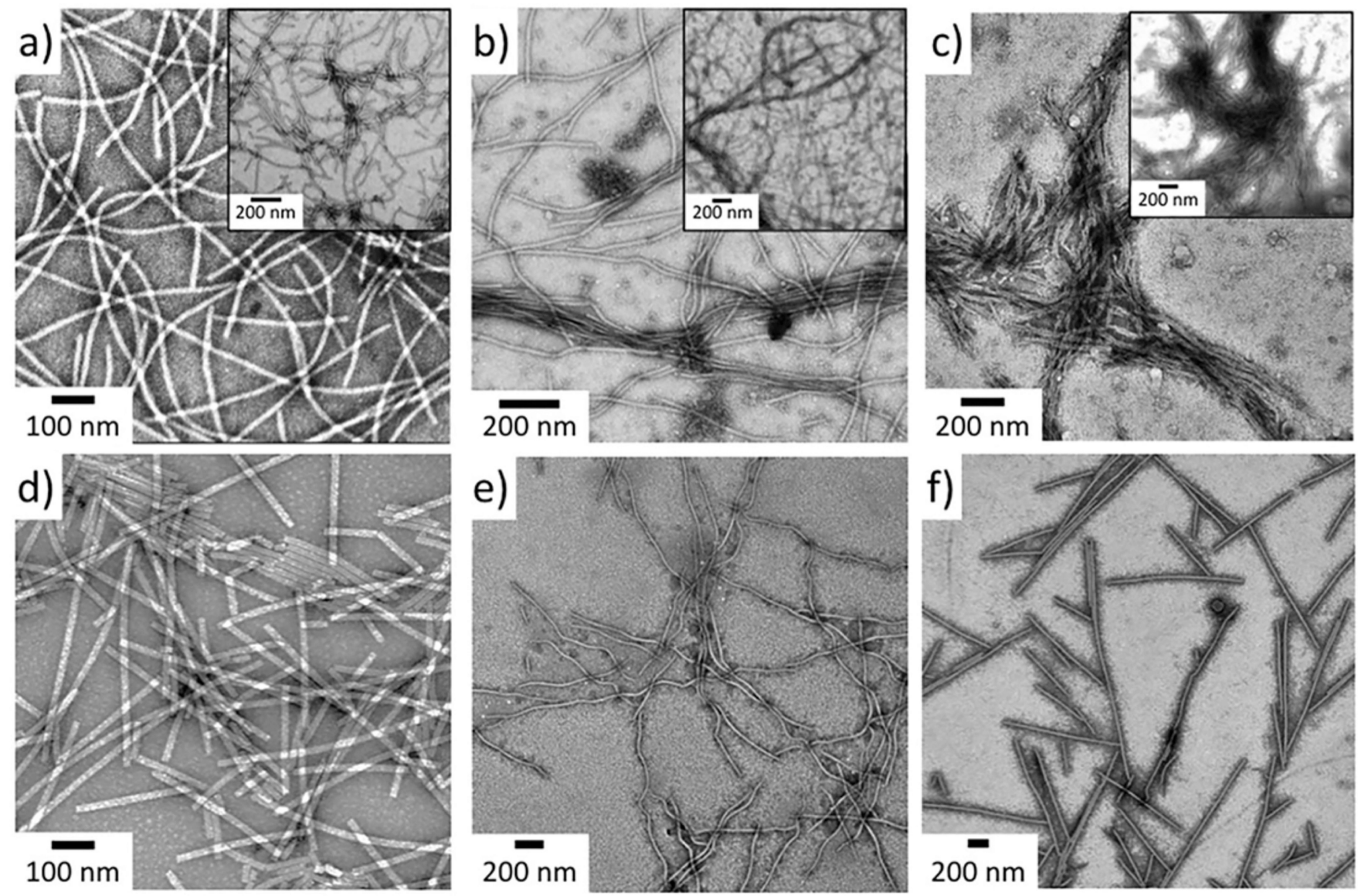

Figure 3.

Aggregation of PVX VNPs by serum in vitro. Filamentous PVX nanoparticles (a) show moderate aggregation with naïve serum (b) but form large highly aggregated clusters when incubated with sera from PVX-primed mice (c). TMV nanorods (d) show no apparent aggregations or clustering when incubated with sera similarly, indicating PVX-specificity of the sera (e and f). 
a)
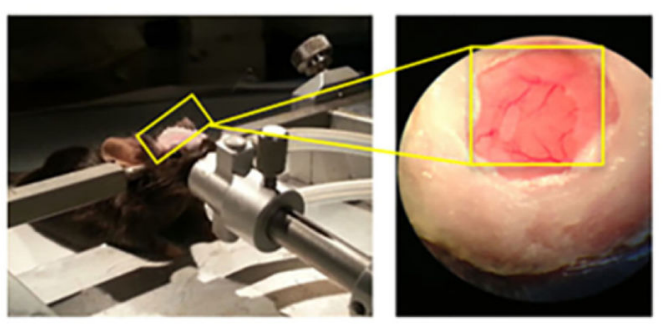

b)

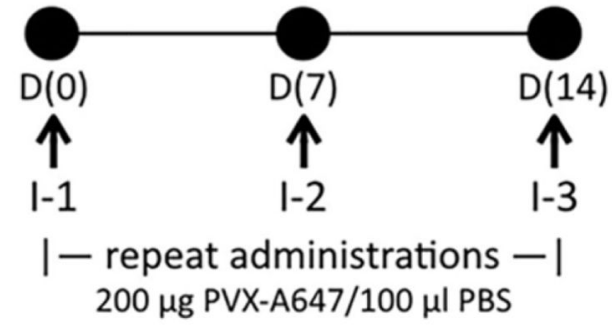

c)

d)

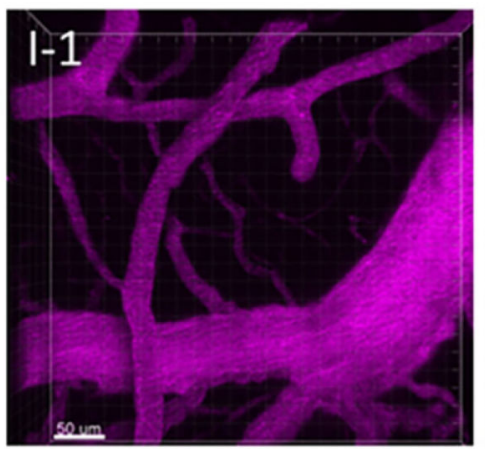

|-1

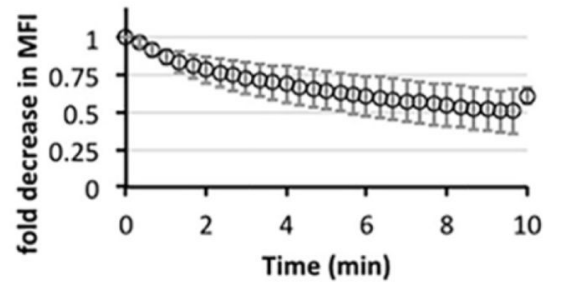

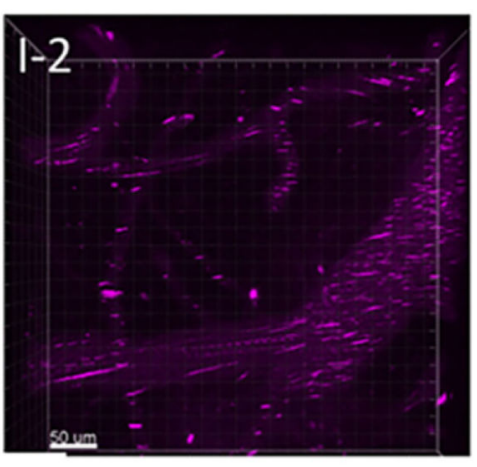

1-2

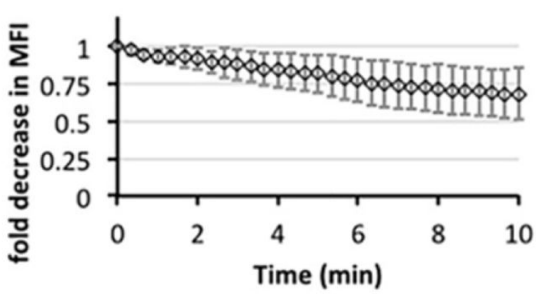

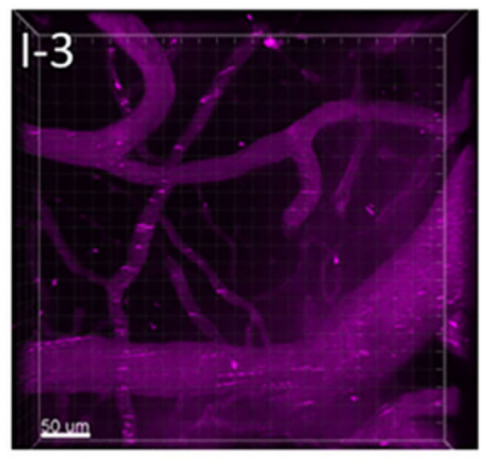

1-3

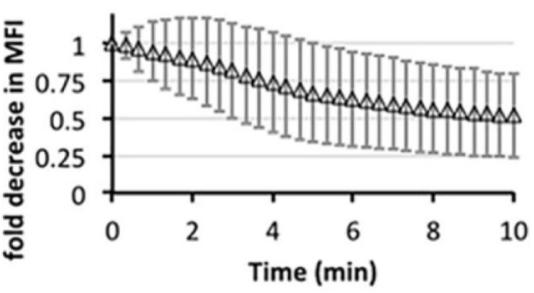

Figure 4.

Intravital imaging for real time monitoring of PVX-A647 in circulation. C57BL/6-Tg(UBCGFP) mice with surgically placed cranial windows were used to image CNS vasculature using 2P-LSM following PVX-A647 injections (a). PVX-A647 was intravenously administered as weekly injections (b). CNS vasculature was imaged continuously for up to 10 min following each injection. Representative image showing a fixed area imaged following successive injections (PVX-A647 is pseudo-colored pink; c). Change in mean fluorescence intensity (MFI; average of three mice) plotted against time following each round of intravenous injection $(\mathrm{d})$. 